ARTICLE

\title{
Nutrition in children with long-term health conditions
}

\author{
A Westwood, MB ChB, DCH (SA), FCPaed (SA), MD \\ School of Child and Adolescent Health, Department of Paediatrics and Child Health, Faculty of Health Sciences, University of Cape Town, and \\ Deparmtent of Paediatrics, New Somerset Hospital, Cape Town, South Africa
}

Corresponding author: A Westwood (anthony.westwood@uct.ac.za)

Long-term health conditions (LTHCs) in children may affect nutrition and growth by means of multiple mechanisms. Both undernutrition and overweight/obesity are risk factors. Direct effects of the condition that may cause undernutrition include increased resting energy expenditure, excess losses through malabsorption, difficulty ingesting food, and decreased appetite. Indirect effects of LTHCs may be mediated by learnt or adaptive behaviours, secondary anorexia, inappropriate diets, or conditions that aggravate existing social nutritional risks to the child. Undernutrition may have significant consequences for the child, including reduced life expectancy. Overweight is a particular risk in children with neurological LTHCs. Regular clinical assessment, including anthropometry, is required to prevent and detect malnutrition. Anticipatory nutritional guidance to the child and caregivers is required and must be adapted to the specific LTHC. Controlling the disease processes that contribute to malnutrition and optimising energy intake are fundamental elements of prevention and management. Interventional feeding regimens, such as surgical approaches, may be required. A mutidisciplinary team, which includes a dietitian, should manage complex LTHCs and LTHC-associated malnutrition.

S Afr Med J 2015;105(7):606. DOI:10.7196/SAMJnew.7784

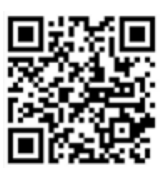

A significant proportion of children in any community have long-term health conditions (LTHCs). This term is preferred to 'chronic disease'. Firstly, chronic has a connotation of severity. Secondly, many children with long-term health problems do not have a disease, as the problem may be a congenital condition or an acquired disability with health consequences. Up to $20 \%$ of children have an LTHC, while $<5 \%$ have a severe LTHC. ${ }^{[1]}$ There is a wide range of conditions that share a set of potential secondary effects mediated by the presence of the condition (regardless of its specific characteristics) and the long duration of evolving childhood.

- For children with LTHCs there is a need for greater involvement with healthcare services than in the case of their peers.

- There are potential effects on neurodevelopment, self-image and self-efficacy, and other psychological functioning.

- There are potential effects on the family.

- There are potential effects on growth and/or nutrition.

Not all of these effects may be present and they may vary in intensity. Many may be preventable by anticipatory interventions. The effects may act synergistically to produce secondary disabilities. This synergistically negative effect is especially important if the LTHC has an intrinsic effect on nutrition and growth; optimal outcomes for a child with an LTHC can only be achieved if optimal growth is achieved. In the absence of an LTHC having an intrinsic effect on growth (e.g. untreated growth hormone deficiency or skeletal disorder), nutrition is the main arbiter of growth.

In many severe conditions, undernutrition is directly linked to life expectancy, even in conditions such as sickle cell disease, ${ }^{[2]}$ which are not always regarded as having a major nutritional aspect. Another consequence of undernutrition - delayed puberty - is associated with problems such as decreased bone mineral density and negative selfimage, which have potential consequences in adult life.

Over- and undernutrition are risks in a wide range of LTHCs. Chronic deficits of micro- or macronutrients can limit somatic growth and have varying direct effects on organ function, depending on the specific LTHC. For example, decreased muscle bulk secondary to undernutrition may limit neurodevelopment in a child with a cardiorespiratory or neurological condition. Overweight can produce secondary disability in these conditions by aggravating any limitation of activity due to the LTHC.

Therefore, special consideration must be given to nutrition for most children with LTHCs as part of any management plan that aims to optimise a child's quality of life.

This article explores the mechanisms by which nutritional problems may occur in LTHCs. It provides pointers to the prevention and management of such problems. Specific micronutrient deficiencies are not discussed.

\section{The normal situation}

It is a fundamental precept of auxology (the study of human growth) that the growth of a developing organism can only occur if energy intake exceeds energy expenditure. Genetic growth potential will only be realised if there is a sufficiently positive energy balance to allow optimal growth.

In healthy children energy is expended as follows:

- Resting energy expenditure (REE) - energy used in metabolic (e.g. protein synthesis, ion pumps) and resting mechanical activities (e.g. heartbeat and breathing) that are necessary to maintain body integrity. This comprises $50-60 \%$ of total energy expenditure (TEE).

- Diet-induced thermogenesis (5 - $8 \%$ of TEE).

- Activities of daily living (30 - 40\% of TEE).

- Faecal energy losses, which vary with dietary energy intake.

Fig. 1 demonstrates the normal energy balance in a healthy child. Inadequate energy intake relative to expenditure limits growth. Excess energy intake relative to expenditure may lead to fat deposition and overweight.

\section{Mechanisms of suboptimal nutrition}

Fig. 2 summarises the many mechanisms that can lead to undernutrition in children with LTHCs; there may be more than one in a 


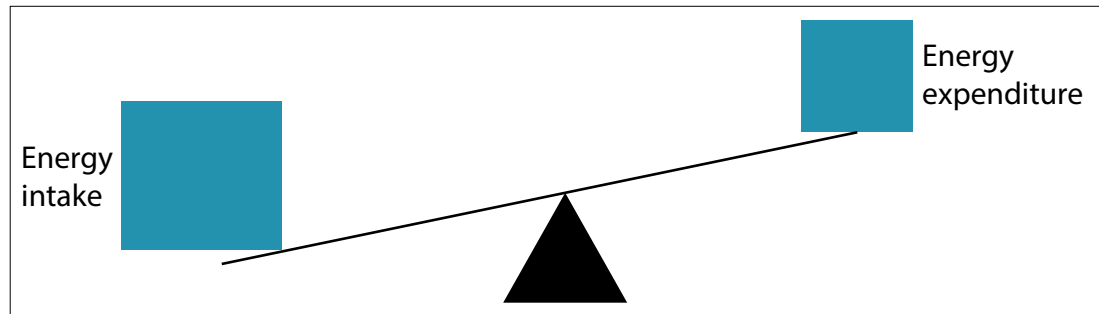

Growth

Fig. 1. Energy balance and growth.

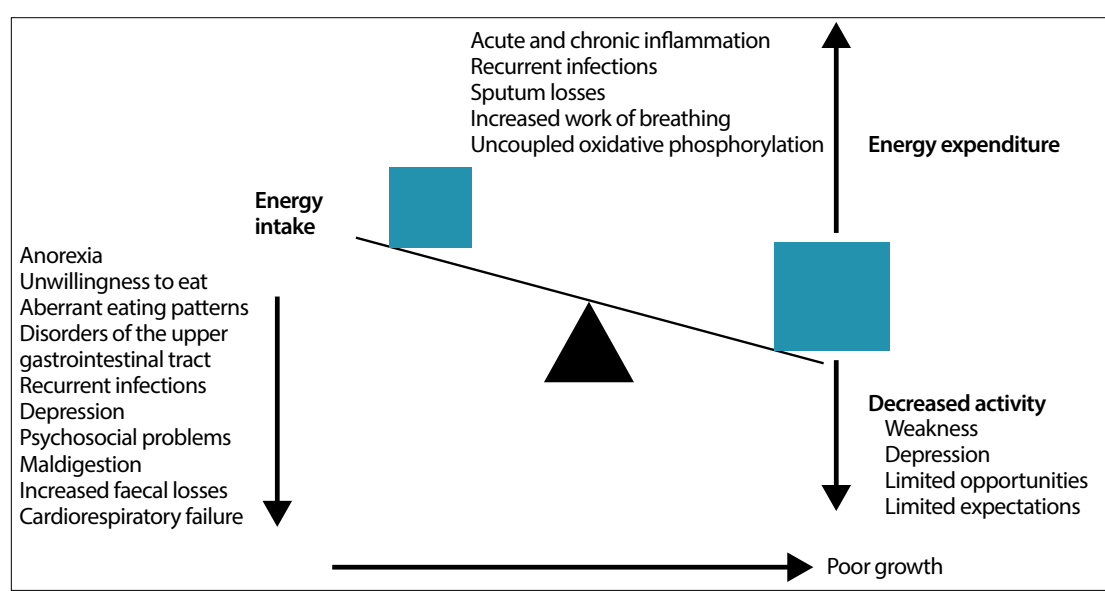

Fig. 2. Mechanisms of negative energy balance in long-term health conditions.

child. When present together, some may be more than additive in their effect, e.g. recurrent infections leading to recurrent anorexia. The potential mitigating energy balance effect of reduced activities of daily living has risks, such as aggravation of depression or anorexia. Not shown in Fig. 2 is the inability to use available nutrient-derived energy, because this contributor to limitation of growth in some LTHCs (especially endocrine disorders such as hypothyroidism) is not mediated through nutrition.

\section{Decreased intake}

\section{Anorexia and decreased appetite}

These are often primary effects in LTHCs affecting the gastrointestinal tract (GIT). The child with primary GIT dysmotility or that which arises secondary to malabsorption in liver, gut or pancreatic diseases learns that eating produces bloating or discomfort and reduces the quantity and frequency of food intake. Chronic renal failure produces primary anorexia. Children with immune deficiencies are prone to opportunistic mouth or oesophageal infections (e.g. thrush), which produce dysphagia. Recurrent infections also reduce appetite for significant periods.

Secondary anorexia may occur owing to micronutrient (especially iron and zinc) deficiencies. These deficiencies may be secondary to poor intake or malabsorption. Uncontrolled perennial allergic rhinitis reduces taste differentiation and appetite. Secondary anorexia also occurs when a child fails to cope with living with an LTHC and becomes depressed. Long-term medication effects on the GIT, such as nausea, vomiting or abdominal pain, may decrease appetite. ${ }^{[3]}$

\section{Unwillingness to eat}

Unwillingness to eat is often seen in patients with conditions such as cystic fibrosis, where high-energy intake is promoted to overcome excess energy losses or energy expenditure. Poorly managed parental zeal produces an oppositional response in the child. This may set a pattern for life, unless it is recognised and dealt with. Children and adolescents with type 1 diabetes mellitus can be adept at limiting intake - which may not be nutritionally optimal - to achieve a thin body habitus.

\section{Inability to eat enough}

Children with congenital or acquired abnormalities of the upper GIT or the mouth, such as cleft palate, are at risk of undernutrition. Likewise, in neurological disorders, such as quadriplegic cerebral palsy, inadequate oral phase of swallowing and inco-ordinate deglutition limit energy intake of liquids and/or solids. Without multidisciplinary interventions in such conditions the risk of undernutrition is high. Cardiorespiratory disorders, such as left-toright congenital heart shunts in small infants, may cause tachypnoea and fatigue that limit the infant's ability to breastfeed or bottle-feed adequately. Chronic anaemia may have the same effect in infancy, e.g. in sickle cell disease.

Therefore, careful attention to nutrient intake and eating habits is required in most children with LTHCs. Additional attention is required in children from economically deprived homes, as reduced intake due to poverty or social disruption has a greater effect on a child with an LTHC than on an otherwise healthy child.

\section{Malabsorption}

While the potential contribution of malabsorption to decreased food energy availability and consequent undernutrition is obvious in GIT conditions such as coeliac disease and obstructive biliary disorders, malabsorption is a factor in many other LTHCs. Recurrent diarrhoea (including undiagnosed giardiasis) is common in immunodeficiencies. Uncontrolled cardiac failure and chronic hypoxaemia affect the absorptive function of the small intestine.

\section{Psychological disorders}

While psychiatric LTHCs such as anorexia nervosa have clear nutritional consequences, other LTHCs may be associated with enough primary or secondary psychopathology to affect energy intake. ${ }^{[3]}$ This is especially the case with depression - often the result of dysfunctional adaptation to the presence of an LTHC - and its consequences for the child and family. Awareness of this risk and screening for its early manifestations should be part of standard clinical care for children with LTHCs.

\section{Inappropriate diets}

Medical practitioners need to be aware that families of children with an incurable or severe LTHC may use dietary remedies to 'cure' or ameliorate the condition. Parents may remove milk from the diet of a child with chronic lung disease because it is perceived to 'increase mucus'. Some LTHCs, such as coeliac disease, require special diets. In such cases the input of a dietitian is necessary to avoid an energy-poor diet.

\section{Increased energy expenditure Increased inflammation}

Diversion of nutrient energy into inflammation is a potent contributor to suboptimal nutrition and growth in many LTHCs. This is an especially important consideration in conditions that render children prone to recurrent or chronic infections. Examples are HIV-associated diseases and chronic 
suppurative disorders of the lung, such as those that occur with chronic Pseudomonas aeruginosa infection in cystic fibrosis. Nutritional losses from chronic sputum production can be significant. Noninfective chronic inflammation in autoimmune disorders may add to the intrinsic risk of underweight and growth restriction caused by musculoskeletal pain and reduced movement.

\section{Increased energy utilisation}

As with inflammation, energy diversion to other non-growth activities occurs in many LTHCs. The most common of these is utilisation of abovenormal amounts of energy for maintenance of oxygenation in congenital and acquired cardiac and respiratory diseases. REE can be $>20 \%$ higher than normal owing to increased cardiorespiratory effort. In some diseases this diversion is not recognised because the level of disease activity is not correctly assessed. A common example of this phenomenon is undertreated asthma. If not compensated for, this diversion will contribute to suboptimal growth and possible overt malnutrition.

In LTHCs such as hyperthyroidism and mitochondrial disorders increased REE is intrinsic to the disease and needs to be compensated for. REE may be raised in some patients with HIV infection despite good disease control.

\section{Prevention and early detection of undernutrition}

Undernutrition should be prevented in children with LTHCs. Clinical assessment of the risk to an individual is an essential part of comprehensive care. Continuity of the healthcare team is an important component, as the risk changes with phases of the child's life and the LTHC. Therefore, attention to nutrition and growth should be a regular and planned part of clinical consultations.

The essential elements of the nutritional assessment include:

- Dietary history.

- Review of eating and mealtime habits.

- Evidence of GIT dysfunction.

- Anthropometric assessment: weight, height, weight-for-height or body mass index, mid-upper-arm circumference (6 months - 5 years).

- Plotting these measures and assessing trends on appropriate growth charts.

- Physical examination.

Measurement of height is especially important in detecting longerterm nutritional effects of LTHCs that may not be acute enough to manifest as overt weight loss or wasting.

Overall evaluation of the status of the LTHC may also raise issues that could result in nutritional depletion if not remedied or compensated for, e.g. frequent infections or disease exacerbations.

Anticipatory guidance is an essential part of long-term care for children with LTHCs. Such guidance is required for nutritional problems and issues of development, mental health and transition to adult-orientated healthcare. The clinician is required to make an assessment of a child's nutritional risk, which is then used to construct a counselling approach. For example, a baby with a leftto-right cardiac shunt or malabsorption syndrome requires more frequent weighing than other infants. Special guidance on energy intake may be required in children with chronic infections. Educators may need to play a role in the child's nutrition during the school day. Advice on how to handle expected eating behavioural patterns may assist a parent with these common challenges. Dietitians can play a crucial role in anticipatory guidance and specific dietary and eating advice. They should be included in the healthcare team at an early stage to assist with children who have an LTHC and a significant risk of undernutrition.

\section{Management of undernutrition}

Most of the principles of management of undernutrition in children are applicable to children with LTHCs. These principles include:

- Controlling the disease processes that contribute to the energy deficit, where possible.

- Optimising energy intake through:

- attention to the form and balance of the diet, including the possible use of supplements

- improving eating behaviour where necessary

- deciding whether an interventional form of feeding is required (discussed below)

- ensuring that energy intake during hospital admissions and disease exacerbations is maximised.

- Use of the World Health Organization 10-step intervention (suitably modified for the specific LTHC) for severely malnourished children.

- Avoiding refeeding syndrome in children with severe malnutrition.

- Regular follow-up and assessment by a multidisciplinary team.

A dietitian is indispensable in nutritional rehabilitation. Expertise in assessing the nature and extent of the problem, constructing appropriate diets, counselling on eating habits and reviewing progress are essential elements in the care of a child with an LTHC and undernutrition. South Africa (SA)'s primary healthcare nutrition National Therapeutic Programme provides supplements to children with LTHCs on the advice of a dietitian employed by the Department of Health. ${ }^{[4]}$

A psychologist may be required if eating behaviours cannot be ameliorated by the standard healthcare team, or if there is a significant psychological component to the causation of the undernutrition.

Speech therapists can assist with feeding and sensory and other problems that lead to limited energy intake. Specialist nurses often play a pivotal role in nutritional management of children with LTHCs in well-resourced countries, but there are very few such nurses in SA. Doctors must not abdicate responsibility for canvassing and co-ordinating such advice and inputs in the comprehensive care of a child.

Interventional feeding regimens may be required, such as specialised bottles for children with cleft lips and palates, and nasogastric or gastrostomy feeds. The last may be used to bypass upper GIT and facial anomalies. Overnight supplementary feeds are valuable for children whose energy requirements are so high that they cannot reasonably be expected to consume enough food and supplements (e.g. in cystic fibrosis), or if their appetite is poor (e.g. in chronic renal failure). All of these interventions require the input of nurses and dietitians, again emphasising the role of the multidisciplinary team in the management of children with LTHCs.

\section{Overweight and obesity}

Problems of overweight are not uncommon among children with LTHCs, especially those with a neurological component. The risk arises from a combination of compensatory eating behaviours and inappropriately high-calorie, low-residue diets, because these are easier for the child to masticate and swallow, and relatively lowenergy use in activities of daily living. Again, prevention is better than cure. Awareness of the risk and anticipatory guidance allow the clinician and healthcare team to work with the parents, family and child to prevent the child from becoming overweight. The dietitian's role can be pivotal. 


\section{Conclusion}

Nutritional problems, especially undernutrition, are probable in many children with LTHCs. These problems arise from the direct effects of the LTHC and the secondary effects common to most LTHCs - often in combination. Awareness is essential and prevention must be an aim of comprehensive care. Management of potential and actual nutritional problems requires a planned approach and includes contributions from many members of a multidisciplinary team over the entire period that an LTHC is present.

\section{References}

1. Westwood T, Robertson A. A child with a long-term health condition. In: Kibel M, Saloojee H, Westwood T. Child Health for All. 5th ed. Cape Town: Oxford University Press, 2012.

2. Al-Saqladi AW, Cipolotti R, Fijnvandraat K, Brabin BJ. Growth and nutritional status of children with homozygous sickle cell disease. Ann Trop Paediatr 2008;28:165-189. [http:// dx.doi.org/10.1179/146532808X335624]

3. Quick VM, Byrd-Bredbenner C, Neumark-Sztainer D. Chronic illness and disordered eating: A discussion of the literature. Adv Nutr 2013;4:277-286. [http://dx.doi.org/10.3945/ an.112.003608

4. Labadarios D, Steyn NP, Mgijima C, Daldla N. Review of the South African nutrition policy 1994 2002 and targets for 2007: Achievements and challenges. Nutrition 2005;21:100-108. [http://dx.doi, org/10.1016/j.nut.2004.09.014 